\title{
Deux carrières : Jean Bène et Paul Coste-Floret
}

In: Pôle Sud, N²2 - 1995. pp. 151-157.

\section{Citer ce document / Cite this document :}

Damamme Dominique. Deux carrières : Jean Bène et Paul Coste-Floret. In: Pôle Sud, N² - 1995. pp. 151-157.

doi : $10.3406 /$ pole. 1995.895

http://www.persee.fr/web/revues/home/prescript/article/pole_1262-1676_1995_num_2_1_895 


\section{Deux carrières : \\ Jean Bène et Paul Coste-Floret}

par D. Damamme

C'est une fin ordinaire des mémoires de DEA que d'être voués à l'oubli. Le second numéro de Pôle Sud est une bonne occasion pour "revisiter" quelques-unes des biographies d'hommes politiques réalisées par les doctorants du CEPEL. En raison des trajectoires contrastées qu'elles retraçaient, on a choisi deux études de "grands" hommes politiques héraultais de la IV République, Jean Bène (Olivier Pelat, J. Bène, Etude d'une carrière politique, 1988) et Paul Coste-Floret (François Vergnes, Paul Coste-Floret, ou la sagesse en politique, 1988), opposés à la fois par leurs propriétés sociales, leurs ressources politiques et leurs représentations du métier politique. Elles présentaient en outre le sérieux avantage d'éclairer le jeu des formes culturelles et des habitudes mentales dans la carrière de ces deux notables.

Né en 1911 à Montpellier, Paul CosteFloret est issu d'une bourgeoisie commerçante et robine. Le père, Jules Coste-Floret, avocat, appartient à l'espèce politique localement rare du catholicisme républicain. Membre du Parti Démocrate Populaire, ami de $\mathrm{R}$. Cornilleau et de Champetier de Ribes, il sera conseiller municipal de Montpellier de 1929 à 1935. "Héritiers" respectueux de l'héritage, les fils CosteFloret, Alfred et Paul - jumeaux, leur histoire est inextricablement mêlée - perpétuent la tradition politique et idéologique paternelle. Le jeune Paul Coste-Floret est inscrit au Cercle Montalembert et à la Conférence Saint-Vincent-de-Paul; à 18 ans, il adhère au Parti Démocrate de l'Abbé Lemire, pour, écrit-il, "faire cohabiter Révolution, République et Évangile". Porte-parole naturel et naturellement porte-parole des catholiques libéraux, il préside l'Association des Étudiants Catholiques de Montpellier, branche de l'Association Catholique de la Jeunesse Française. C'est à cette époque qu'il participe, aux côtés de P. Boulet ', à la brève expérience de Sud (1930-1933), quotidien catholique des partisans de la République qui cherche à s'ouvrir un espace entre L'Éclair royaliste et le Petit Méridional radical-socialiste. Les deux frères font leur droit ${ }^{2}$. Filiation oblige. Mais, à la différence de leur père, ils optent pour l'enseignement. En 1937, Paul Coste-Floret est reçu à l'agrégation de droit public alors qu'Alfred, l'autre lui-même, l'inséparable rival, lui échoue à ce même concours. Ce n'est ni le premier ni le dernier épisode de la longue course, moitié-poursuite, moitié-relais, entre les deux frères. Alfred, devenu Maître de Conférences à la Faculté de droit de Strasbourg se lie à René Capitant et participe à la fondation du mouvement de résistance "Combat". C'est lui qui met Paul, en poste à Alger, en rapport avec R. Capitant quand ce dernier s'y fait nommer en 1941. Paul Coste-Floret 


\section{Pôle Sud}

s'engage alors à son tour dans la Résistance, et cet engagement - qui ira, selon le témoignage de quelques proches, jusqu'à la lutte armée - constitue le départ de sa longue carrière politique nationale.

A Alger, Paul devient conseiller technique d'André Philip, puis directeur de cabinet de François de Menthon, ministre de l'Intérieur. A la Libération, il est nommé Procureur général-adjoint à Nuremberg auprès d'Edgar Faure. Alfred, Maître des Requêtes au Conseil d'État, sollicité par le MRP pour se porter candidat aux élections législatives, décline l'offre et c'est Paul, décidément voué à la sphère publique, qui est investi. Élu député MRP de l'Hérault en 1945 , il retrouve son siège en 1946 , 1951,1956 et en 1958 sous l'étiquette "Union Nationale Républicaine et de Défense Viticole". Sous la IVe, il est ministre pratiquement sans interruption de 1947 à 1954 (Ministre de la Guerre, 1947, de la France d'Outre-Mer, 1947-1948, Ministre de l'Information, 1952, Ministre d'État chargé de la Réforme constitutionnelle en 1953, Ministre de la Santé 19531954). Parallèlement, il cherche à s'implanter localement, en faisant fructifier la dot de sa femme dont le père, A. Railhac, a été député radical et maire de Lodève. Non sans difficulté : en 1949, il est battu aux cantonales par une alliance des socialistes et des communistes qui vise à l'empêcher d'accéder au conseil général (canton de Ganges). S'il devient maire de Lamalou-lesBains en 1953, il est encore battu aux cantonales de 1955 (canton de Saint-Gervais). En 1959, il se présente aux municipales de Lodève, où il est élu. En 1967, son opiniâtreté est récompensée puisqu'il entre au conseil général, mais sa carrière de "professionnel" de la politique va se terminer cette année-là. D'abord, après une défaite aux législatives de 1962, il est à nouveau battu en 1967 et en 1968. Ensuite, une accusation de fraude au scrutin municipal le conduit à démissionner de son mandat de maire de Lodève. Il ne lui reste plus que son mandat de conseiller général et la mairie de Lamalou que gère par procuration sa femme. Dans la mesure où la preuve de la responsabilité personnelle de $P$. Coste-Floret n'a jamais pu être apportée, sa démission rappelle que le maintien dans le jeu politique menace parfois de détruire non seulement le crédit politique mais aussi l'identité sociale qu'il est préférable, dès lors, d'abandonner une activité qui risque de vous dégrader. Pendant quelques années, P. Coste-Floret reprend ses enseignements de droit. Nommé en 1971 membre du Conseil Constitutionnel, il est élu après son mandat au décanat de la Faculté de droit (1976) puis Président de l'Université de Montpellier I (1977). Il meurt deux ans plus tard, en 1979.

Il n'est pas besoin d'épiloguer longuement sur les causes politiques et institutionnelles des échecs législatifs de P. CosteFloret : favorisé par le scrutin proportionnaliste de la IV ${ }^{e}$ République, le centrisme démocrate-chrétien a buté sur le scrutin majoritaire de la $V^{c}$ et sur la double concurrence du gaullisme - P. Coste-Floret ayant refusé de se rallier au général De Gaulle - et de la gauche. Léchec de Paul Coste-Floret est celui d'une force partisane mais il traduit aussi les difficultés d'enracinement local d'un type social et d'un style d'action. Bourgeois, pratiquant la représentation politique sans 


\section{Deux carrières : J. Bène et P. Coste-Floret}

s'identifier à ses mandants, juriste ${ }^{3}$, universitaire, amateur de politique, c'est-à-dire, dans sa conception, d'idées, faisant profession d'amateurisme, il n'était sans doute pas prêt à se plier aux contraintes du métier politique local ${ }^{4}$, ni même peut-être capable de s'y adapter. Incapable de maîtriser certains des savoir-faire politiques requis, il refuse de s'adonner aux formes de communication politique localement les plus valorisées 5. La politique pour Coste-Floret, c'est d'abord le domaine de la décision : homme de dossiers, homme de cabinet, d'écriture, il manifeste un goût pour la gestion qui lui vaut une réputation de "froideur" dans les rapports humains. Pour ce lettré, la politique c'est aussi le débat d'idées : en témoignent ses interventions au conseil général et plus encore ses "Tribunes Libres" du Midi-Libre 6 . Mais cette conception abstraite de la politique qu'il tient de son métier de professeur, de "donneur de leçons", le font taxer d' "intellectuel". On peut penser que c'est à ce décalage entre l'homme et le territoire, autant que la fragilité de sa famille politique, qu'est dû son échec local. Sur les quatre registres symboliques qui structurent l'action politique locale, le registre politique (idéologique), le registre de la compétence (conformité au modèle valorisé du maire gestionnaire), le registre de la proximité sociale (conformité aux groupes sociaux) et le registre local (conformité aux valeurs locales), ces deux derniers registres lui manquaient. Bref, il n'a pas réussi ou n'a pas voulu se conformer à la représentation socialement dominante de l'élu local. Faut-il s'étonner si en raison de la nature de ses ressources et du capital politique qu'il avait accumulé et si du fait de sa position dominée, il ait été tenté, à partir des années soixante, d'incarner une figure de sagesse ${ }^{7}$.

Pour appartenir au pouvoir périphérique ${ }^{8}$, un homme politique peut être plus central que bien des acteurs nationaux. Un rôle de second n'est pas nécessairement un second rôle. Ce fut le cas de Jean Bène, maire de Pézenas de 1932 à 1941, de 1945 à 1947 , puis de 1953 à 1977, conseiller général de 1945 à 1979, Président du conseil général de l'Hérault de 1945 à 1979, Sénateur de 1946 à 1971 (année où il se démet de ses mandats de maire et de sénateur ${ }^{9}$ ) exemple typique du notable qu'on dit "local", en dépit de ses fonctions politiques et du fait qu'il partageât son temps à part égale entre Paris et l'Hérault.

Fils unique d'un courtier en vin de Pézenas ${ }^{10}$, d'une famille de petite bourgeoisie, portée à investir dans l'École et marquée à la fois par le moralisme républicain et le catholicisme ${ }^{11}$, J. Bène franchit sans trébucher les barrières scolaires qui donnent un vrai titre de bourgeoisie. Au terme de ses études de droit, il obtient un double diplôme d'études supérieures de droit pénal et de science administrative et financière - signe de ses projets politiques? - et devient avocat à Pézenas, le premier de sa profession, indice là encore de ses ambitions politiques locales mais aussi d'une sorte de "timidité" sociale qui le conduit à des choix prudents, à rester en territoire connu. J. Bène ou jusqu'où ne pas aller trop loin.

Inscrit à la SFIO, il est très vite et très jeune, à 28 ans, désigné tête de liste aux élections municipales de 1929. La liste SFIO 


\section{Pôle Sud}

est battue par la liste du Parti Radical mais Bène entre au conseil municipal. En 1931 il est élu conseiller de l'arrondissement de Béziers, puis en 1932, à la suite d'une série de crises internes au conseil municipal, il se fait élire maire de Pézenas où il applique les recettes du socialisme municipal ${ }^{12}$.

Dans le champ politique local, Bène est un tard-venu. S'il a pu montrer à Pézenas son savoir-faire politique et s'il bénéficie du soutien d'E. Barthe et de son journal $L e$ Devoir Socialiste ${ }^{13}$, il ne reste plus de place pour lui à l'échelon de la députation. Les élections de 1932 ont porté à la Chambre un socialiste, Léon Baylet, puis en 1936, d'autres "jeunes" encore, Roucayrol et J. Moch, proche de L. Blum, que les majoritaires ont envoyé se faire élire dans l'Hérault, au même moment où la Fédération de l'Hérault passe sous le contrôle de Bataille Socialiste ${ }^{14}$, courant dont il dénonce le "sectarisme". "La SFIO, écrit-il alors, c'est le parti du peuple, mais le parti républicain du peuple". Le socialisme de Bène, c'est celui de la République sociale, pas celui de Marceau Pivert. De 1932 à 1940 , les voies politiques, partisanes et électorales, sont ainsi obstruées ${ }^{15}$. Bref, la carrière de Bène est bloquée et toutes les conditions sont réunies pour qu'il reste confiné à sa position de petit élu local.

La guerre change radicalement l'économie des positions politiques et partisanes. Destitué par Vichy en 1941, J. Bène entre dans la Résistance, à "Combat" ${ }^{16}$. Chef départemental des MUR, puis du MLN, il est à la fin de la guerre à la fois responsable local de la SFIO clandestine et président du Comité Départemental de Libération
(CNR), disposant d'une triple légitimité - "historique", élective, partisane - qui l'autorise à incarner localement la légalité républicaine $^{17}$ : c'est lui qui fait office puis qui seconde le Commissaire de la République (qu'il refuse d'être), nomme sous-préfets et procureurs, qui installe les conseils locaux de libération à la place des anciens conseils municipaux.

Même s'il cumule alors des sources de légitimité qui dépassent de loin son territoire, le retour à l'ordre pose à nouveau la question de la division du travail au sein du parti socialiste. Le poids local du PCF, notamment dans le Biterrois, la présence de Jules Moch à Sète conduisent en 1945 à exclure Bène de la représentation parlementaire. A Jules Moch, la députation ${ }^{18}$, à Charles Alliès la gestion du parti, à J. Bène le territoire. Il le gardera.

J. Bène illustre, par sa biographie, un double idéal-type de notable, notabilisation politique par accumulation de capital symbolique ${ }^{19}$ et notabilisation politico-administrative, saisissable notamment au travers de sa position politique départementale (sénateur-président du conseil général) et sa participation à de multiples organismes publics départementaux et nationaux : Conseil Supérieur des Alcools, Commission Nationale des Secteurs Sauvegardés, Commission Supérieure de l'Aménagement Rural, Président de la Société d'Aménagement du Département de l'Hérault et unique élu membre de la Mission interministérielle d'Aménagement du Languedoc-Roussillon.

Sans être spécifique au Languedoc, puisqu'on la repère également en Bretagne ${ }^{20}$ et qu'elle dépend pour partie des stratégies 


\section{Deux carrières: J. Bène et P. Coste-Floret}

utilisées par les élites politiques locales dans la reproduction de leur domination politique, la notabilisation par territorialisation trouve, au delà de la rhétorique de l'intérêt général mise en lumière par P. Grémion, dans les traits spécifiques de structure sociale et la culture politique locale des conditions et des formes sur lesquelles les entreprises politiques peuvent adosser leur offre de représentation. Si la fonction notabiliaire découle d'une position de négociateur entre la société locale et l'État, ses formes apparaissent liées à une capacité à incarner une histoire et un territoire, c'està-dire une entité qui n'est pas synonyme de circonscription administrative, qui ne se réduit pas en un agrégat d'intérêts matériels, mais qui renvoie et rappelle une identité culturelle. Ce qui se comprend facilement si l'identité politique territoriale a une consistance historique. Ce type de notabilisation a contribué à la constitution d'un réseau de notables et à des pratiques systématiques de compromis et de collusion ${ }^{21}$. Intercesseur, le notable est pourvoyeur de biens, biens nullement abstraits, publics et privés, collectifs et individuels. $\mathrm{Si}$ la fonction implique une pratique clientélaire, encore faut-il disposer de biens qui intéressent, ou aussi accepter de s'y livrer : il n'est pas certain que P. Coste-Floret ait eu les moyens de mettre en place un clientélisme rentable ni, d'ailleurs, que ce type de pratique fit partie, à ses yeux, de la politique légitime : il n'entrait ni dans son éthique ni simplement dans l'éthos d'un homme sans "territoire".

Médiateur, le notable local l'est doublement, entre sa communauté et l'administration, mais aussi entre la communauté locale et la communauté nationale, l'État, les autres, les "ils". Mais plusieurs manières de construire les enjeux et les clivages sont possibles. A l'instar de Paul Coste-Floret ou des élus communistes, l'entrepreneur politique aura tendance à politiser les rapports sociaux quand il occupe une position politiquement dominée ou, en cas de panne, dans les rapports collusifs. La caractérisation du notable par l'apolitisme (c'est la position sociale qui est déterminante), la personnalisation (c'est l'hérédité, la tradition familiale qui font la notoriété) et le localisme (c'est la défense quasi corporative d'intérêts locaux qui est l'essentiel de l'activité de l'élu) ${ }^{22}$, retraduit d'abord des traits typiques d'une position dominante. Précisément, il y a l'autre manière de politiser, par la mobilisation du clivage société locale/État, le localisme et l'intercession notabiliaire conduisant à surpolitiser le couple interne/externe. Le radicalisme languedocien, puis le socialisme de la SFIO paraît bien une expression de ce localisme là. Sans substantifier ce type de clivage, on ne peut que relever la diversité des contextes d'opération de la thématique et la fréquence de ses incarnations : "résistance cathare", "dont il subsiste partout des racines", ou encore la résistance de Montmorency contre Richelieu et du "pays" contre Pétain ${ }^{23}$.

A travers le prisme des carrières publiques de Paul Coste-Floret et de Jean Bène, l'analyse des conditions du métier politique local rappelle, encore une fois, l'attention qu'il faut prêter, au delà de la différence des histoires et des pratiques des acteurs, à leur rencontre avec le territoire. 


\section{Pôle Sud}

\section{NOTES}

1. Dirigeant local de l'A.C.J.F., membre du Sillon, fondateur de Jeune République dans l'Hérault, il deviendra maire de Montpellier en 1936 (et à nouveau en 1945 sous l'étiquette du Rassemblement Républicain et Socialiste). Il sera exclu du MRP en 1950.

2. Mais aussi leur sœur cadette, Mme Furet, professeur de droit public à la faculté de droit de Montpellier.

3. B. Gaïti, De la Quatrième à la Cinquième République. Les conditions de la réalisation d'une prophétie, Thèse Paris I, 1992

4. Cf. à l'inverse l'article de P. Secondy sur René Couveinhes. De même, à la différence de Jean Bène, $\mathrm{P}$. Coste-Floret n'a pas véritablement travaillé à construire une implantation territoriale solide en intégrant sur un même territoire tous les échelons électifs. On peut penser aussi que, du fait de son poids politique national, sa candidature concentrait sur elle l'ensemble des opposants au "système".

5. Métier politique et communication, Centre de Recherches politiques de la Sorbonne, Paris I, 1990 ; Politix, n² 27 : Le métier d'élu, 1994.

6. Dont il est depuis la Libération administrateur, poste qui traduit les alliances politiques nationales et locales à partir de la rupture du tripartisme. En 1947, Jean Bène, directeur politique et administratif du journal, reprend, grâce à Jacques Soustelle alors Ministre de l'Information, le contrôle du Midi-Libre dirigé par les communistes. Le conflit avec l'équipe de la rédaction du journal se termine par un partage des actions, $32 \%$ à J. Bène et Jules Moch, $32 \%$ au MLN, $32 \%$ aux journalistes, le reste au Parti Radical.

7.F. G. Bailey, Les règles du jeu politique, Paris, PUF, 1971, notamment sur les tâches du leader et les positions d'arbitre et de médiateur qui, par extrapolation, permettent de réfléchir à une position de sage en politique.

8. P. Grémion, Le Pouvoir périphérique, Paris, Seuil, 1976.

9. Il est battu aux élections cantonales de 1979 par P. Guirault, alors inscrit au PCF.

10. Il naît en 1901.

11. Alors que la branche paternelle de la famille Bène est "socialisante", la mère de J. Bène est catholique pratiquante. Comme le sera également sa femme, institutrice. "Si j'ai pu au cours de ma très longue vie politique avoir quelques initiatives heureuses, soutenir quelques causes d'intérêt public, m'attacher à défendre la liberté des plus défavorisés, en réalité je n'en ai aucun mérité car j'ai été élevé par des parents si désintéressés, si respectueux des convictions des autres, si soucieux du bien être général, si scrupuleux en ce qui concerne les deniers publics, que les habitudes de ma jeunesse étant prises, il m'aurait été impossible de m'en évader" (J. Bène, 1979).

12. En 1932, la SFIO atteint $36 \%$ des suffrages exprimés dans le département.

13. Le Devoir Socialiste de Barthe plus tard soutiendra Vichy.

14. Sur les luttes internes à la SFIO, $c f$. J. Sagnes, Politique et syndicalisme en Languedoc, Montpellier, Université Paul Valéry, 1986.

15. O. Dedieu me signale que J. Bène, à cette époque, est dans une position d'héritier en attente d'héritage, dauphin d'André, le Conseiller général SFIO de Pézenas et directeur du Devoir Socialiste, dauphin également de Léon Baylet, le député de la circonscription. En 1945, si Jean Bène laisse en 1945 "naturellement sa place à J. Moch comme premier de liste" (C. Alliès), la SFIO croit assurer son élection en le plaçant en second sur la liste.

16. Il sera Michel à Pézenas, Garaud en tant que chef de l'AS, Azéma comme chef de mission du BCRA de Londres. A la fin de la guerre, il dirige un maquis dans le secteur de Saint-Pons. M. Sadoun, Les socialistes sous l'occupation, Paris, PFNSP, 1982.

17. Face à l'épuration qu'il canalise et à quelques velléités communistes.

18. Jean Bène laisse en 1945 "naturellement sa place à J. Moch comme premier de liste" (C. Alliès). 


\section{Deux carrières : J. Bène et P. Coste-Floret}

19. J.-L. Briquet, "La gestion familiale d'un patrimoine politique, le cas des grands notables en Corse depuis la IIIe République", Colloque de l'AFSP : Héritage politique et parenté, Dijon, 1991. M. Offerlé, "Usages et usures de l'hérédité en politique", RFSP, n 5, 1993.

20. S. Berger, Les paysans contre la politique, Paris, Seuil, 1975.

21. Qui se retraduit dans la tradition de "courtoisie" des débats du conseil général et de l'exclusion des "polémiques" politiques. "Dans la fonction de Président du conseil général, ma tâche a été tellement facilitéE par la courtoisie de mes collèges" (J. Bène, 1979).

22. P. Alliès, "Europe et territoire : vers un nouveau localisme", Quaderni, n 13-14, p. 119.

23. J. Bène, Midi-Libre, 5 Novembre 1968. 